\title{
Condiciones óptimas del análisis CASA-Mot del semen de verraco: efecto de la tasa de fotogramas para diferentes cámaras y campos de recuento espermático
}

\author{
Optimal conditions for CASA-Mot analysis of boar semen: Effect of frame \\ rate for different chambers and sperm count fields
}

\author{
Vinicio Barquero', Francisco Sevilla', Josué Calderón-Calderón', Mónica Madrigal- \\ Valverde $^{2,3}$, Marlen Camacho', Hernán Cucho ${ }^{4}$, Anthony Valverde ${ }^{1,5}$
}

\section{Resumen}

El objetivo del estudio fue determinar el efecto de la tasa de fotogramas sobre diferentes profundidades de cámara de recuento espermático en semen de verraco y analizar la distribución de los espermatozoides en los campos longitudinales de la cámara de conteo. Se colectó una muestra de semen a 10 verracos Pietrain de 2-3 años. Se determinaron ocho parámetros de cinética espermática mediante el sistema CASA (Computer-assisted semen analysis)-Mot (ISAS ${ }^{\circledR} \mathrm{v} 1$ ). Se utilizaron cámaras ISAS ${ }^{\circledR} \mathrm{D} 4 \mathrm{C}$ con profundidades de 10, 16 y $20 \mu \mathrm{m}$ a una frecuencia de captura de imagen de 25,40 y 60 fotogramas por segundo (fps). La profundidad de cámara afectó las variables de cinética espermática, obteniéndose valores más altos a $20 \mu \mathrm{m}$. La tasa de fotogramas (FR) presentó una relación positiva con los parámetros de cinética obteniéndose valores más altos a $60 \mathrm{fps}$, excepto para la amplitud del desplazamiento lateral de la cabeza (ALH) que presen-

${ }^{1}$ Instituto Tecnológico de Costa Rica (ITCR), Escuela de Agronomía, Campus Tecnológico Local San Carlos, Laboratorio de Reproducción Animal, BIOTEC, Alajuela, Costa Rica

${ }^{2}$ Universidad de Costa Rica (UCR), Escuela de Zootecnia, Ciudad Universitaria Rodrigo Facio, San Pedro, Montes de Oca, San José, Costa Rica

${ }^{3}$ Área Académica del Doctorado en Ciencias Naturales para el Desarrollo, Instituto Tecnológico de Costa Rica. CTLSC, Alajuela, Costa Rica

${ }^{4}$ Universidad Nacional de San Antonio Abad del Cusco, Escuela Profesional de Zootecnia, Cusco, Perú

${ }^{5}$ E-mail: anvalverde@tec.ac.cr

Recibido: 7 de marzo de 2021

Aceptado para publicación: 18 de septiembre de 2021

Publicado: 27 de octubre de 2021

CLos autores. Este artículo es publicado por la Rev Inv Vet Perú de la Facultad de Medicina Veterinaria, Universidad Nacional Mayor de San Marcos. Este es un artículo de acceso abierto, distribuido bajo los términos de la licencia Creative Commons Atribución 4.0 Internacional (CC BY 4.0) [https:// creativecommons.org/licenses/by/4.0/deed.es] que permite el uso, distribución y reproducción en cualquier medio, siempre que la obra original sea debidamente citada de su fuente original 
tó un comportamiento inverso. Según el análisis de regresión no lineal, la variable más sensible al cambio en FR fue la velocidad curvilínea (VCL), donde el mejor nivel fue dado con 80 fps. Se observó una disminución progresiva en los patrones cinemáticos conforme los espermatozoides avanzaban por los campos de las cámaras. Se concluye que el uso de diferentes cámaras de recuento y el incremento en el número total de fps genera cambios significativos en la estimación de parámetros de cinética espermática en el verraco.

Palabras clave: espermatozoide, fotograma, cámara de recuento, verraco, reproducción

\section{Abstract}

The aim of the study was to determine the effect of the frame rate on different depths of the sperm counting chamber in boar semen and to analyse the distribution of sperm in the longitudinal fields of the counting chamber. A semen sample was collected from ten 2-3-year-old Pietrain boars. Eight sperm kinetic parameters were determined using the CASA (Computer-assisted semen analysis) - Mot (ISAS ${ }^{\circledR}$ v1) system. ISAS ${ }^{\circledR}$ D4C cameras with depths of 10,16 and $20 \mu \mathrm{m}$ were used at an image capture rate of 25,40 and 60 frames per second (fps). Chamber depth affected the sperm kinetic variables, obtaining higher values at $20 \mu \mathrm{m}$. The frame rate (FR) presented a positive relationship with the kinetic parameters, obtaining higher values at $60 \mathrm{fps}$, except for the amplitude of the lateral displacement of the head (ALH), which presented opposite values. According to the nonlinear regression analysis, the most sensitive variable to the change in FR was the curvilinear velocity (VCL), where the best level was observed with $80 \mathrm{fps}$. A progressive decrease in kinematic patterns was provided as sperm advanced through the fields of the chambers. It is concluded that the use of different counting chambers and the increase in the total number of fps generates significant changes in the estimation of sperm kinetics parameters in the boar.

Key words: spermatozoa, frame, counting chamber, boar, reproduction

\section{INTRODUCCIÓN}

La eficiencia en el uso de las técnicas de la reproducción porcina es esencial y forma parte del desafío que enfrenta la industria a futuro (Broekhuijse et al., 2015). Asimismo, la fertilidad puede estar asociada a múltiples factores del verraco, como el manejo, el intervalo de extracción (Lopez-Rodriguez et al., 2017), la concentración (Valverde et al., 2019d), la edad y la movilidad (Broekhuijse et al., 2012). Por lo tanto, el estudio de estos factores y los parámetros de la calidad del semen forman parte del avance tecnológico empleado a partir del análisis espermático por medio de los sistemas computarizados de análisis seminal (CASA, Computer Assisted Semen Analysis) (Valverde et al., 2019b, 2020).

Los sistemas CASA permiten analizar el semen de forma objetiva y reducir el error asociado respecto de los análisis realizados en campo (Amann y Waberski 2014; Gallagher et al., 2018; Valverde y MadrigalValverde, 2018) y aportan más información al describir patrones cinemáticos de los espermatozoides (Ehlers et al., 2011; Valverde y Madrigal-Valverde, 2018). El análisis profundo de estos parámetros cinéticos junto con la movilidad seminal permite una mejor caracterización de los criterios de evaluación de los reproductores (Barquero et al., 
2021); sin embargo, la determinación de los valores de movilidad puede variar incluso cuando se utilizan configuraciones similares entre los sistemas CASA(Ehlers et al., 2011). Además, las variables de movilidad y cinética están influenciadas por el tipo de equipo utilizado y sus aditamentos, como por ejemplo, las cámaras de recuento espermático (Soler et al., 2012). En estudios realizados con diferentes tipos de cámara de recuento, profundidad y/o alturas y formas de llenado, se ha demostrado el efecto sobre algunos parámetros de movilidad (Del Gallego et al., 2017; Soler et al., 2017, 2018). Incluso los campos de análisis dentro de una misma cámara de recuento pueden influir sobre las variables cinemáticas de los espermatozoides (Valverde y Madrigal-Valverde, 2019).

Los sistemas CASA-Mot presentan variaciones debido a factores internos y externos al software (Valverde et al., 2020). Un factor importante de considerar en el análisis es la tasa de fotogramas (FR) utilizada (Valverde et al., 2019c). Hay evidencia que el FR no condiciona los resultados de movilidad total y progresiva (Castellini et al., 2011). En el análisis cinemático, las trayectorias de las células pueden variar según la configuración de fotogramas por segundo (frame per second, fps) (Lu et al., 2014). De esta manera, las diferentes configuraciones de los sistemas CASA-Mot pueden condicionar los análisis cinéticos, ya sea por un inadecuado reconocimiento de la distancia en las trayectorias de las células espermáticas analizadas (Amann y Waberski, 2014) o por limitaciones técnicas de las cámaras digitales (Boryshpolets et al., 2013; Bompart et al., 2018; Caldeira et al., 2019; Gacem et al., 2020b). Con base en estos antecedentes, el objetivo del presente estudio fue determinar el efecto de la tasa de fotogramas sobre diferentes cámaras de recuento espermático para semen de verraco analizado con un sistema CASA-Mot, así como analizar la distribución de los espermatozoides a lo largo de los campos longitudinales de la cámara de recuento.

\section{Materiales y MéTodos}

\section{Localización}

Se trabajó con cuatro granjas porcinas en los cantones de Río Cuarto y San Carlos, de la región Huetar Norte, Costa Rica. Las características climáticas de la zona durante el periodo experimental indicaron una temperatura ambiental en el rango de 16$28{ }^{\circ} \mathrm{C}$ (mínima y máxima), humedad relativa de $70-90 \%$ y una precipitación anual superior a $3000 \mathrm{~mm}$. El manejo productivo de las granjas se encontró dentro de las regulaciones del Reglamento sobre granjas porcinas N. ${ }^{\circ}$ 37155-MAG (Ministerio de Agricultura y Ganadería, Costa Rica). Los análisis seminales se realizaron en el Laboratorio de Reproducción Animal del Centro de Investigación y Desarrollo en Agricultura Sostenible para el Trópico Húmedo (CIDASTH), Campus Tecnológico Local San Carlos, Instituto Tecnológico San Carlos, Alajuela, Costa Rica. El experimento fue realizado durante el año 2017.

\section{Animales}

Se utilizaron 10 verracos (Sus scrofa domestica), a razón de $2.5 \pm 1.0$ verracos por granja, con edades entre 2 y 3 años y con un adecuado estado de salud. Los animales pertenecían a una línea comercial de la raza Pietrain que se selecciona genéticamente por caracteres de crecimiento y rendimiento magro. Los animales se alojaron en corrales individuales dentro de un sistema de producción intensivo con acceso a agua ad libitum y una oferta total de $2.0 \mathrm{~kg} \mathrm{día}^{-1}$ (distribuido en dos partes) de alimento balanceado fabricado en la granja, conforme a los requerimientos nutricionales para porcinos (National Research Council, 2012).

\section{Semen}

Un técnico recolectó un eyaculado por verraco $(n=10)$ mediante el método de «mano enguantada» utilizando un termo colector y 
una bolsa de colecta seminal (Minitube, Alemania) que separaba la secreción gelatinosa de las glándulas bulbouretrales del eyaculado. La muestra seminal se protegió de la luz y se colocó en un baño maría a $37^{\circ} \mathrm{C}$. El semen se diluyó 1:1 (vol:vol) con un diluyente comercial (Androstar ${ }^{\mathbb{P} P l u s, ~ M i n i t u b e, ~ A l e m a-~}$ nia) pudiendo mantenerse hasta por siete días. Las muestras, al analizarse en el laboratorio, debían presentar un mínimo de $85 \%$ de morfología normal, $85 \%$ de movilidad total (ISAS ${ }^{\circledR} v 1$, Proiser R+D, España) y una concentración superior a $250 \times 10^{6}$ espermatozoides $\mathrm{ml}^{-1}$ (Fotómetro SDM 1, Minitube, Alemania); además, se verificó que estuvieran libre de contaminación por medio de una evaluación macroscópica de color y olor.

Las muestras se colocaron en una caja térmica de polipropileno $\left(17^{\circ} \mathrm{C}\right.$; Plastimex, México) para su traslado hasta el laboratorio, donde se sometieron al protocolo de ingreso a las instalaciones y se conservaron durante $24 \mathrm{~h}$ en posición horizontal a $17^{\circ} \mathrm{C}$ (Polar Refrigeration, Reino Unido). Posteriormente, las muestras se homogenizaron y se tomó una alícuota de $1 \mathrm{ml}$ en un microtubo (Eppendorf $^{\circledR}$, Alemania) de $1.5 \mathrm{ml}$ que fue colocada en una estufa a $37^{\circ} \mathrm{C}$ durante 20 min antes del análisis espermático. Luego, se tomaron dos alícuotas de $400 \mu 1$ en microtubos Eppendor $\mathrm{f}^{\circledR} \mathrm{de} 1.5 \mathrm{ml}$ (réplicas) y se diluyeron en una proporción de 1:5 (vol:vol) de semen y diluyente, respectivamente, para su análisis.

\section{Cámaras de recuento y técnica de llenado}

El análisis de las variables de cinética espermática se realizó mediante el uso de cámaras de recuento comerciales ISAS ${ }^{\circledR} \mathrm{D} 4 \mathrm{C}$ que poseen un cubreobjetos fijo unido por pegamento (Proiser R+D, España) con profundidades de 10,16 y $20 \mu \mathrm{m}$, cuyo principio de llenado es la difusión capilar. Las cámaras se precalentaron a $37^{\circ} \mathrm{C}$ en una placa calefactora (ISAS PC $12 \mathrm{~T}^{\circledR}$, Proiser R+D, España). Las muestras se homogenizaron

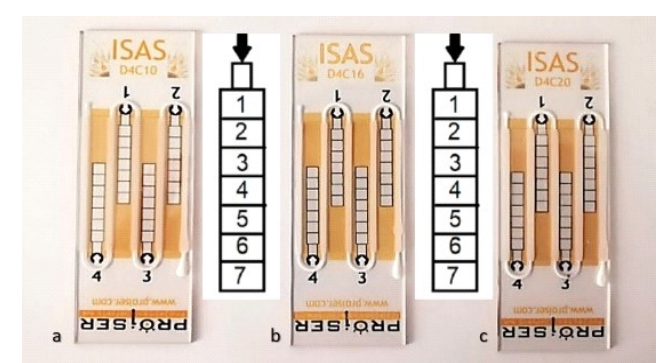

Figura 1. Diferentes profundidades de cámara de recuento ISAS ${ }^{\circledR} \mathrm{D} 4 \mathrm{C}$ desechables y el campo analizado. (a): D4C10. (b): D4C16. (c): D4C20. Los números (1-7) dentro de la cámara representan los campos de captura

antes de cargarse en las cámaras de recuento. El cargado $(2.7 \mu \mathrm{l})$ se realizó siguiendo las recomendaciones del fabricante (Figura 1). Las cámaras se mantuvieron en la placa calefactora durante $15 \mathrm{~s}$ a $37^{\circ} \mathrm{C}$ para evitar el choque térmico. Para el análisis, se asignó al azar el orden de las cámaras utilizadas para evitar el efecto del tiempo de incubación. Cada muestra fue evaluada en las tres cámaras por duplicado. Para estudiar el efecto de la ubicación del campo sobre la movilidad de los espermatozoides se identificaron siete campos longitudinalmente en D4C 10, 16 y $20 \mu \mathrm{m}$ (Figura 1) y se capturó un mínimo de 200 espermatozoides por campo de análisis.

\section{Análisis CASA-Mot}

La evaluación objetiva de la cinética de los espermatozoides se realizó utilizando el sistema computarizado CASA-Mot ISAS ${ }^{\circledR} \mathrm{v} 1$ (Proiser R+D, España) combinado con un microscopio UB200i (Chongqing UOP Photoelectric Technology, China) equipado con un objetivo de contraste de fase negativo 10X (AN 0.25), una platina calefactora a $37^{\circ} \mathrm{C}$ incorporada y una cámara digital de Proiser 782m (Proiser R+D). Las muestras fueron analizadas utilizando una frecuencia de captura de imagen de 25, 40 y 60 fps capturando un total de 25 imágenes. 
Los parámetros CASA-Mot fueron generados utilizando el módulo de análisis de movilidad (ISAS ${ }^{\circledR} \mathrm{v} 1$ ) con la configuración para la especie porcina recomendada por el fabricante, capturándose siete campos por cámara, con una réplica en cada caso. El análisis de los patrones cinemáticos de los espermatozoides consiste en un proceso de captura inicial de una imagen que va a proporcionar un centroide para cada espermatozoide en el primer fotograma de una captura y a continuación, en imágenes sucesivas, la localización de la célula a partir del centroide más probable es deducida.

Se evaluaron los siguientes parámetros cinemáticos:

- Velocidad curvilínea (VCL, $\mu \mathrm{m} \mathrm{s}^{-1}$ ). La suma de las distancias entre cada posición del espermatozoide (centroide) dividida por el tiempo de análisis,

- Velocidad rectilínea (VSL, $\mu \mathrm{m} \mathrm{s}^{-1}$ ). La distancia en línea recta entre la primera y la última posición del espermatozoide dividida por el tiempo de análisis,

- Velocidad de trayectoria promedio(VAP, $\left.\mu \mathrm{m} \mathrm{s}^{-1}\right)$. La velocidad promedio de la trayectoria determinada como la velocidad promediada en el tiempo de un espermatozoide a lo largo de su trayectoria media,

- Índice de oscilación (WOB, \%). Oscilación de la trayectoria real alrededor de la trayectoria media. $\mathrm{WOB}=\mathrm{VAP} /$ VCL*100, adimensional,

- Índice de rectitud (STR, \%). Medida de la linealidad de la trayectoria media: STR $=\mathrm{VSL} / \mathrm{VAP} * 100$, adimensional),

- Índice de linealidad (LIN, \%). Linealidad de la progresión hacia adelante o la linealidad de la trayectoria curvilínea. LIN $=\mathrm{VSL} / \mathrm{VCL} * 100$, adimensional

- Desplazamiento lateral de la cabeza $(\mathrm{ALH}, \mu \mathrm{m})$. Distancia promedio de la cabeza de los espermatozoides (centroides) expresada como desviación de la trayectoria media,
- Frecuencia de entrecruzamiento (BCF, $\mathrm{Hz}$ ). Se expresa como el número de movimientos oscilatorios laterales de la cabeza del espermatozoide (centroide) alrededor de la trayectoria media.

Los errores de reconocimiento de las trayectorias los revisó el mismo técnico y los eliminó cuando fue necesario, para evitar la introducción de distorsiones en los resultados finales.

\section{Tasa de Fotogramas}

La tasa de fotogramas óptima se determinó a partir de la VCL de los espermatozoides utilizando las reconstrucciones de las trayectorias punto por punto para cada tasa de fotogramas probada. Los resultados cinéticos se sometieron a un análisis de regresión no lineal mediante el siguiente modelo: $y=\beta . \alpha \exp (-\hat{\mathrm{a}} / x)$, donde $y$ es la VCL, $x$ es la tasa de fotogramas, $\alpha$ es el nivel asintótico, $\beta$ es la tasa de incremento a la asíntota y $\exp$ es la base de los logaritmos naturales.

El significado biológico de la fórmula es que los valores asintóticos para $\alpha$ representan el máximo alcanzable cuando la tasa de fotogramas (frame rate, FR) está por encima del valor umbral. El valor umbral se calculó convencionalmente como el frame rate necesario para obtener el $95 \%$ del valor máximo. La tasa de aproximación a la asíntota representa la dependencia de la curva con el FR; es decir, un valor relativamente mayor de $\alpha$ indica un mayor aumento de la VCL a medida que aumenta el FR y viceversa.

\section{Análisis Estadístico}

El efecto profundidad de la cámara de recuento espermático ISAS ${ }^{\circledR} \mathrm{D} 4 \mathrm{C}(10,16,20$ $\mu \mathrm{m})$ o de la tasa de fotogramas capturado en cada campo de análisis CASA-Mot $(25,40$, $60 \mathrm{fps}$ ) se analizaron bajo un modelo lineal de 
efecto fijo. Las diferencias entre medias se analizaron mediante la prueba de Bonferroni. El modelo estadístico utilizado fue: $Y_{\mathrm{i}(\mathrm{k})}=\mu+$ $\mathrm{C}_{\mathrm{i}}+\varepsilon_{\mathrm{i}(\mathrm{k})}$ o $Y_{\mathrm{i}(\mathrm{k})}=\mu+\mathrm{F}_{\mathrm{i}}+\varepsilon_{\mathrm{i}(\mathrm{k})}$, donde $Y_{\mathrm{i}(\mathrm{k})}=$ representa la variable cinemática de los espermatozoides; $\mu$ es la media general de la variable $\mathrm{Y} ; \mathrm{C}_{\mathrm{i}}=\mathrm{el}$ efecto de la cámara de recuento $(i=1,2,3) ; \mathrm{F}_{i}=$ el efecto de la tasa de fotogramas $(j=1,2,3)$ y $\varepsilon_{\mathrm{i}(\mathrm{k})}=$ el error experimental. Se suponen variables aleatorias e independientes con distribución $N(0, \sigma)$. Todos los análisis se ejecutaron con el programa estadístico InfoStat/P (Di Rienzo et $a l ., 2020)$, con un nivel de significancia de 0.05 . Los resultados se muestran como media \pm error estándar de la media.

\section{Resultados}

Efecto de la profundidad de la cámara de recuento y la tasa de fotogramas utilizada sobre la cinemática de los espermatozoides

El análisis de los parámetros cinemáticos de los espermatozoides (VCL, VSL, VAP, STR, ALH y BCF) fue generalmente caracterizado por un valor significativamente más alto en la cámara de $20 \mu \mathrm{m}$, seguido de la cámara con la profundidad de $16 \mu \mathrm{m}$ y los valores más bajos se asociaron con la profundidad de cámara de $10 \mu \mathrm{m}(\mathrm{p}<0.05)$. No hubo diferencias en la LIN de las cámaras de 10 y $16 \mu \mathrm{m}$, pero sí entre estas respecto de la cámara de $20 \mu \mathrm{m}$. Respecto de la WOB, no hubo diferencias entre las cámaras de 16 y $20 \mu \mathrm{m}$, pero sí hubo diferencias con la cámara de $10 \mu \mathrm{m}$ (Cuadro 1).

Al analizar la cinética espermática en función de la tasa de fotogramas, se encontraron diferencias $(\mathrm{p}<0.05)$ conforme se incrementa el FR para las variables VCL, LIN, STR, WOB, ALH y BCF. El incremento no fue significativo para las variables VSL y VAP después de $40 \mathrm{fps}$, pero los valores obtenidos a 25 fps fueron diferentes respecto de 40 y $60 \mathrm{fps}(\mathrm{p}<0.05)$ (Cuadro 2). Al aumentar el FR se redujo la distancia entre centroides (Figura 2) y con ello disminuyó la distancia de desviación de la cabeza de los espermatozoides a partir de la trayectoria media.

Nivel óptimo de tasa de fotogramas y distribución de las variables cinemáticas en cámaras de recuento espermático a diferentes profundidades

Se calculó el nivel óptimo de tasa de fotogramas para cada profundidad de cámara de recuento. La variación en los resultados indicó un aumento en la VCL al incrementar el FR, indistintamente de las profundidades de la cámara. Sin embargo, el incremento asintótico para la VSL y VAP con el aumento del FR no es tan evidente cuando se utilizan cámaras de recuento de $20 \mu \mathrm{m}$. Los valores de VCL fueron de $40.7 \pm 0.4 \mu \mathrm{m} \mathrm{s}^{-1} \mathrm{a}$ cuando se capturaron 25 fps en cámaras a $10 \mu \mathrm{m}$ de profundidad y $83.5 \pm 2.2 \mu \mathrm{m} \mathrm{s}^{-1}$ cuando se tomaron 60 fps en cámaras a $20 \mu \mathrm{m}$, lo que significó un aumento del $105.1 \%$. En este sentido, la tasa de cambio porcentual para la variable VSL al pasar de 25 a $60 \mathrm{fps}$ en cámaras a $20 \mu \mathrm{m}$ fue de $1.0 \%$ y para el VAP de $12.6 \%$ cuando se compararon los valores obtenidos a 25 y 60 fps en cámaras D4C10 y D4C20, respectivamente (Figura 3).

La variable más sensible a los cambios en el FR fue la VCL. La estimación del nivel óptimo de FR fue superior a $80 \mathrm{fps}$, y la tasa de incremento $(\alpha)$ disminuyó en el orden del $56.5 \%$ al incrementarse en $10 \mu \mathrm{m}$ la profundidad de la cámara de recuento utilizada (Cuadro 3).

Efecto del campo de captura analizado dentro de la cámara de recuento espermático

Cuando se capturaron las imágenes a 25 fps en cámaras de 10 y $16 \mu \mathrm{m}$ no se observó una tendencia clara en los parámetros cinemáticos a medida que los espermatozoides se movilizaron desde el punto de deposición inicial hasta el último campo. Sin embargo, con la D4C10 se observó una mayor $(\mathrm{p}<0.05)$ 
Cuadro 1. Efecto de la profundidad de la cámara de recuento espermático ISAS ${ }^{\circledR} \mathrm{D} 4 \mathrm{C}(10$, $16,20 \mu \mathrm{m})$ sobre variables de cinética celular (medias $\pm \mathrm{EE}$ ) del semen de verracos Pietrain analizado con un sistema CASA-Mot

\begin{tabular}{lccc}
\hline \multirow{2}{*}{ Variable } & \multicolumn{3}{c}{ Profundidad de la cámara de recuento $(\mu \mathrm{m})$} \\
\cline { 2 - 4 } & 10 & 16 & 20 \\
\hline VCL $\left(\mu \mathrm{m} \mathrm{s}^{-1}\right)$ & $52.5 \pm 0.6^{\mathrm{c}}$ & $60.1 \pm 0.5^{\mathrm{b}}$ & $69.8 \pm 0.8^{\mathrm{a}}$ \\
$\operatorname{VSL}\left(\mu \mathrm{m} \mathrm{s}^{-1}\right)$ & $34.0 \pm 0.5^{\mathrm{c}}$ & $37.7 \pm 0.3^{\mathrm{b}}$ & $41.0 \pm 0.4^{\mathrm{a}}$ \\
$\operatorname{VAP}\left(\mu \mathrm{m} \mathrm{s}^{-1}\right)$ & $40.3 \pm 0.6^{\mathrm{c}}$ & $42.7 \pm 0.4^{\mathrm{b}}$ & $47.3 \pm 0.4^{\mathrm{a}}$ \\
LIN $(\%)$ & $71.5 \pm 0.5^{\mathrm{b}}$ & $71.8 \pm 0.4^{\mathrm{b}}$ & $74.9 \pm 0.4^{\mathrm{a}}$ \\
STR $(\%)$ & $79.4 \pm 0.7^{\mathrm{c}}$ & $87.8 \pm 0.2^{\mathrm{b}}$ & $89.6 \pm 0.2^{\mathrm{a}}$ \\
WOB $(\%)$ & $76.4 \pm 0.6^{\mathrm{b}}$ & $78.1 \pm 0.4^{\mathrm{a}}$ & $78.5 \pm 0.5^{\mathrm{a}}$ \\
ALH $(\mu \mathrm{m})$ & $1.4 \pm 0.0^{\mathrm{c}}$ & $1.6 \pm 0.0^{\mathrm{b}}$ & $1.8 \pm 0.0^{\mathrm{a}}$ \\
BCF $(\mathrm{Hz})$ & $10.0 \pm 0.2^{\mathrm{c}}$ & $11.7 \pm 0.1^{\mathrm{b}}$ & $12.6 \pm 0.2^{\mathrm{a}}$ \\
\hline
\end{tabular}

VCL: velocidad curvilínea; VSL: velocidad rectilínea; VAP: velocidad promedio; LIN: índice de linealidad; STR: índice de rectitud; WOB: índice de oscilación; ALH: desplazamiento lateral de la cabeza; BCF: frecuencia de entrecruzamiento

$a, b, c$ Dentro de fila, valores con diferente superíndice presentan diferencias significativas $(p<0.05)$

Cuadro 2. Efecto de la tasa de fotogramas capturado en cada campo de análisis CASA-Mot sobre variables de cinética espermática (medias \pm EE) del semen de verracos Pietrain

\begin{tabular}{lccc}
\hline \multirow{2}{*}{ Variable } & \multicolumn{3}{c}{ Tasa de fotogramas } \\
\cline { 2 - 4 } & 25 & 40 & 60 \\
\hline VCL $\left(\mu \mathrm{m} \mathrm{s}^{-1}\right)$ & $50.7 \pm 0.2^{\mathrm{c}}$ & $59.4 \pm 0.5^{\mathrm{b}}$ & $72.2 \pm 1.0^{\mathrm{a}}$ \\
VSL $\left(\mu \mathrm{m} \mathrm{s}^{-1}\right)$ & $34.5 \pm 0.3^{\mathrm{b}}$ & $38.7 \pm 0.4^{\mathrm{a}}$ & $39.5 \pm 0.5^{\mathrm{a}}$ \\
VAP $\left(\mu \mathrm{m} \mathrm{s}^{-1}\right)$ & $39.2 \pm 0.3^{\mathrm{b}}$ & $45.1 \pm 0.5^{\mathrm{a}}$ & $46.0 \pm 0.6^{\mathrm{a}}$ \\
LIN $(\%)$ & $71.1 \pm 0.3^{\mathrm{c}}$ & $72.2 \pm 0.4^{\mathrm{b}}$ & $75.0 \pm 0.6^{\mathrm{a}}$ \\
STR $(\%)$ & $82.7 \pm 0.4^{\mathrm{c}}$ & $85.7 \pm 0.5^{\mathrm{b}}$ & $88.4 \pm 0.5^{\mathrm{a}}$ \\
WOB $(\%)$ & $76.1 \pm 0.4^{\mathrm{c}}$ & $77.8 \pm 0.4^{\mathrm{b}}$ & $79.1 \pm 0.6^{\mathrm{a}}$ \\
ALH $(\mu \mathrm{m})$ & $1.8 \pm 0.0^{\mathrm{a}}$ & $1.5 \pm 0.0^{\mathrm{b}}$ & $1.5 \pm 0.0^{\mathrm{c}}$ \\
BCF $(\mathrm{Hz})$ & $8.0 \pm 0.1^{\mathrm{c}}$ & $10.7 \pm 0.1^{\mathrm{b}}$ & $15.6 \pm 0.2^{\mathrm{a}}$ \\
\hline
\end{tabular}

VCL: velocidad curvilínea; VSL: velocidad rectilínea; VAP: velocidad promedio; LIN: índice de linealidad; STR: índice de rectitud; WOB: índice de oscilación; ALH: desplazamiento lateral de la cabeza; BCF: frecuencia de entrecruzamiento

$a, b, c$ Dentro de fila, valores con diferente superíndice presentan diferencias significativas $(p<0.05)$ 


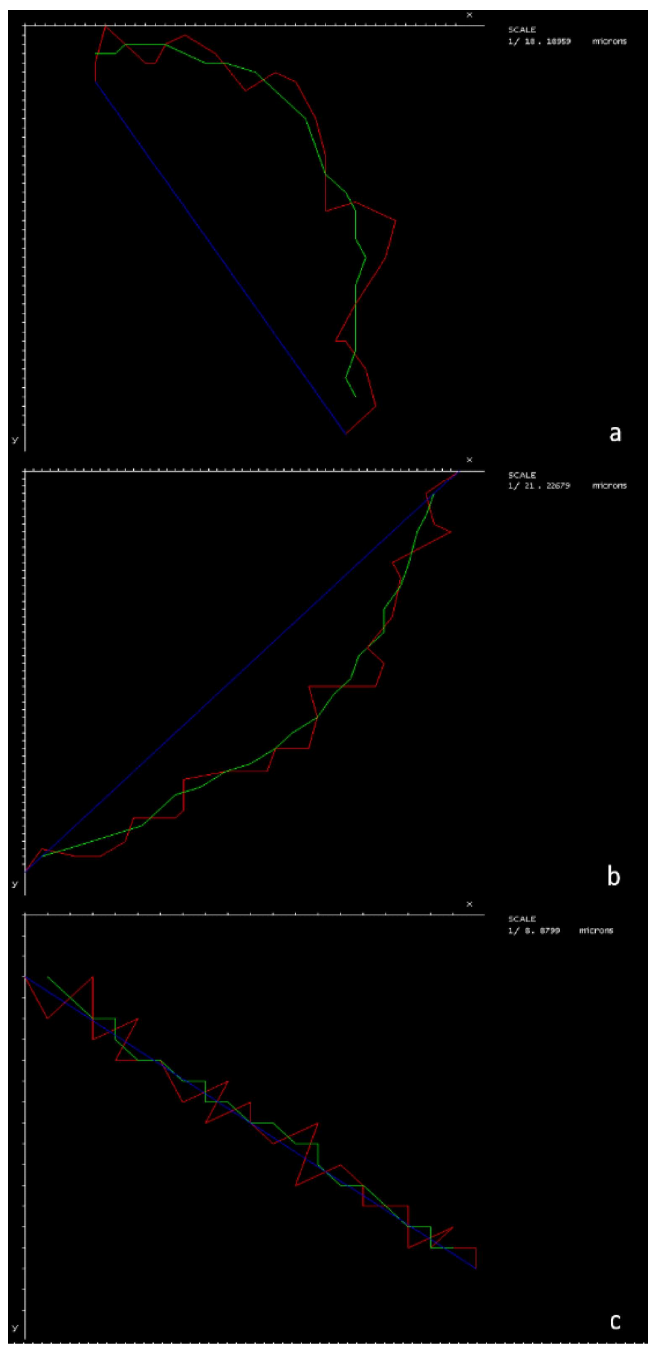

Figura 2. Efecto de la tasa de fotogramas (frame rate) sobre las trayectorias de espermatozoides de verracos Pietrain con la cámara de recuento ISAS ${ }^{\circledR}$ D $4 C 20$. (a): 25 fps. (b): 40 fps. (c): 60 fps. La línea roja representa la velocidad curvilínea (VCL, $\mu \mathrm{m} \mathrm{s}^{-1}$ ), la azul representa la velocidad rectilínea $\left(\mathrm{VSL}, \mu \mathrm{m} \mathrm{s}^{-1}\right.$ ) y la verde representa la velocidad promedio (VAP, $\mu \mathrm{m} \mathrm{s}^{-1}$ )

amplitud del desplazamiento lateral de la cabeza (ALH) en el último campo de análisis. Asimismo, la frecuencia de entrecruzamiento $(\mathrm{BCF})$ con la cámara $\mathrm{D} 4 \mathrm{C} 16$ fue mayor en el último campo de la cámara respecto del primero (Cuadro 4). Con la cámara D4C20 se encontraron los mismos resultados; no obstante, se puede observar un cambio en el patrón de rectitud del espermatozoide a lo largo del proceso de conteo y una reducción en el valor de velocidad (VCL) en el último campo de análisis (Cuadro 4).

Con respecto a la captura de imágenes a $40 \mathrm{fps}$ en la cámara D4C10, los valores más altos de las velocidades (VCL, VAP) y de linealidad (LIN) se observaron más cerca del punto de deposición. En la cámara D4C16, se observó este mismo comportamiento para la VCL y el índice de oscilación (WOB). Sin embargo, el ALH y la BCF mostraron un comportamiento inverso al presentar mayores valores cinemáticos en el último campo de análisis. No hubo diferencias significativas para los valores cinemáticos en los tres campos de análisis en las cámaras D4C20 (Cuadro 5).

Finalmente, cuando se capturaron campos a $60 \mathrm{fps}$, se observó una disminución $(p<0.05)$ de la velocidad (VAP) en el último campo de análisis respecto del punto de deposición en la cámara D4C10. Para D4C16, se observó una disminución $(\mathrm{p}<0.05)$ progresiva de la velocidad (VCL, VSL y VAP) conforme los espermatozoides avanzaban por la ruta de conteo (campos 1 a 7 ), en tanto que para $\mathrm{D} 4 \mathrm{C} 20$ se presentó una variación que contrastó con las observaciones previas, pues la VCL y WOB aumentaron conforme se avanzaba por el campo de análisis (Cuadro 6).

Aunque no hubo un efecto cuadrático significativo en la superficie de respuesta estimada, se tuvo un efecto lineal $(p<0.05)$. Los valores más altos para todos los parámetros cinemáticos, salvo la $\mathrm{BCF}$, se encuentran en la esquina superior derecha de las gráficas de superficie de respuesta, y coincide con los valores más altos de FR y profundidad de cámara de recuento espermático. En forma similar, los valores más bajos de los parámetros cinemáticos, excepto la $\mathrm{BCF}$, se encontraron en la esquina inferior izquierda, que coinciden con un menor FR y menores profundidades de cámara. Para la $\mathrm{BCF}$, los valores más altos de esta variable se encon- 


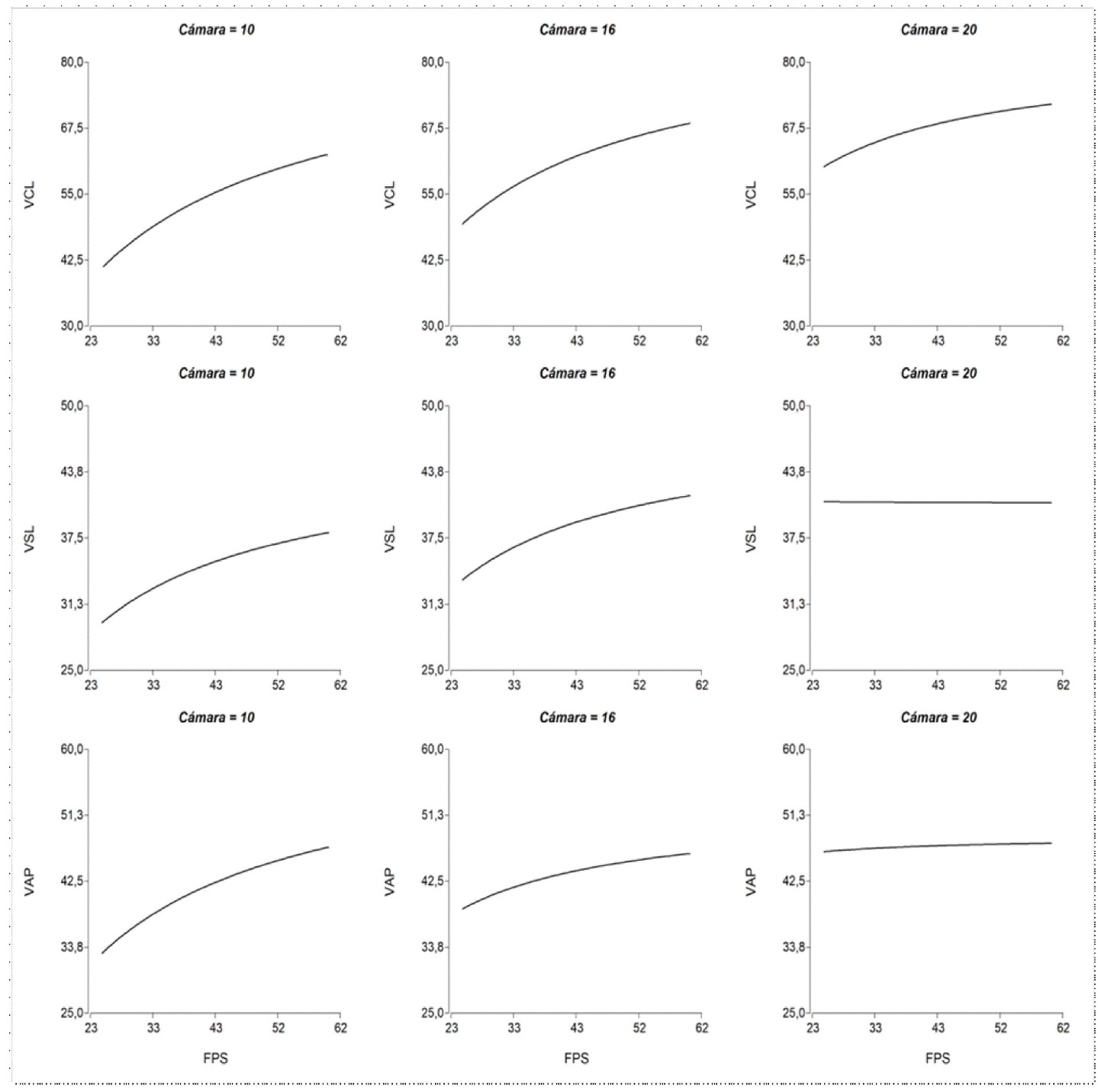

Figura 3. Efecto de la tasa de fotogramas (frame rate) sobre los valores de velocidad del semen de verracos Pietrain determinados a diferentes profundidades de cámara de recuento ISAS ${ }^{\circledR}$ D4C $(10,16$ y $20 \mu \mathrm{m})$. Velocidad curvilínea (VCL, $\left.\mu \mathrm{m} \mathrm{s}^{-1}\right)$, velocidad rectilínea (VSL, $\mu \mathrm{m} \mathrm{s}^{-1}$ ), velocidad promedio (VAP, $\mu \mathrm{m} \mathrm{s}^{-1}$ )

traron cuando la tasa de fotogramas fue menor, pero considerando mayores profundidades de cámara de recuento (Figura 4).

\section{Discusión}

Los resultados del presente estudio pueden explicar la influencia de distintos factores internos y externos que se relacionan con los análisis seminales por medio de los sistemas CASA (Valverde et al., 2020), como por ejemplo, el número y velocidad de fotogramas (Caldeira et al., 2019; Gacem et al., 2020a), el tipo y profundidad de la cámara de recuento (Bompart et al., 2019), la distribución de los campos de análisis en la cámara (Soler et al., 2012) y los algoritmos de análisis seminal del sistema (Urbano et al., 2017; Gallagher et al., 2018). 

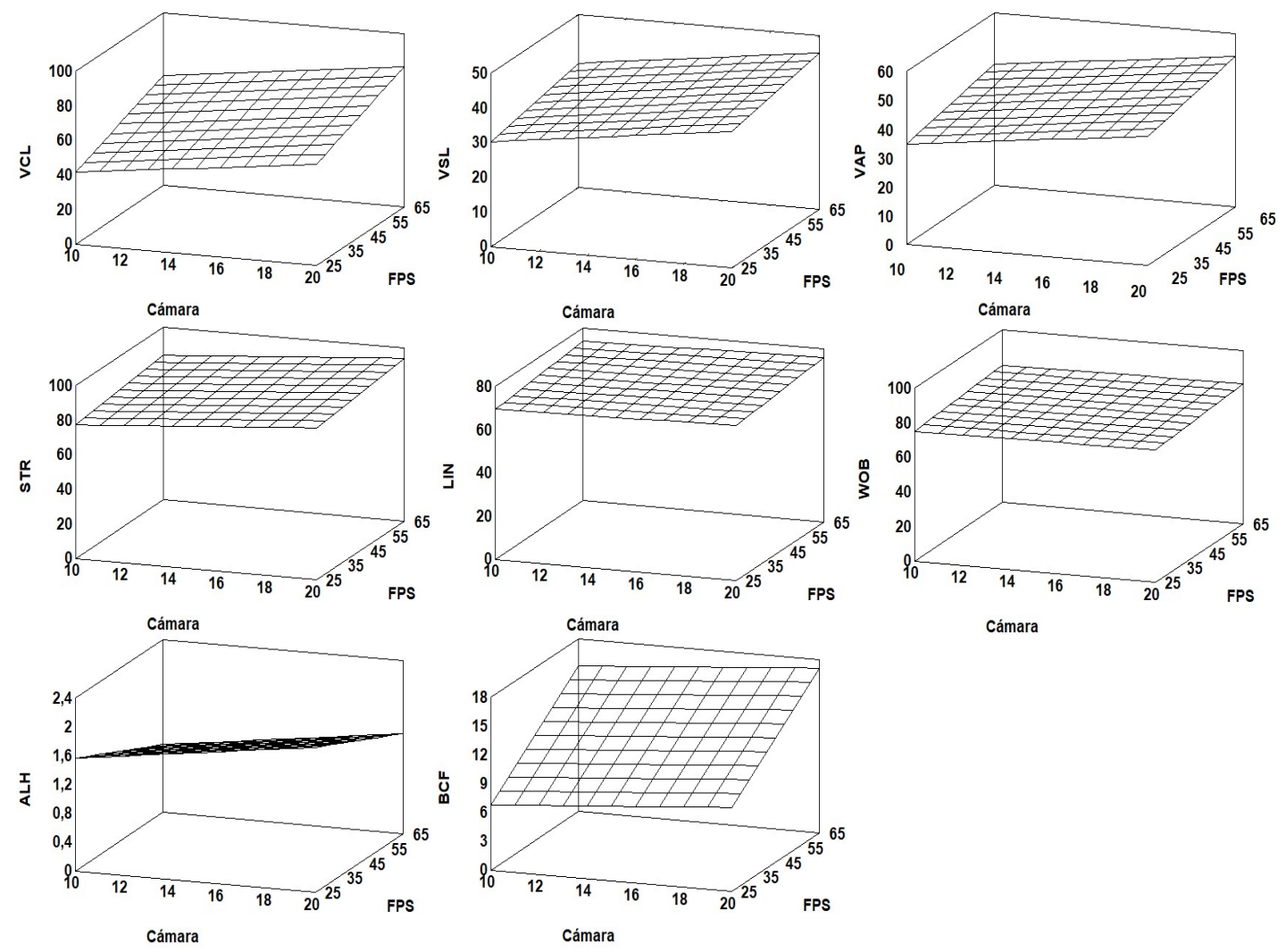

Cámara

Figura 4. Gráficos de superficie de respuesta de profundidad de cámara vs fotogramas por segundo (frames per second, fps) para variables de cinética espermática de verracos Pietrain. VCL: velocidad curvilínea $\left(\mu \mathrm{m} \mathrm{s}^{-1}\right)$; VSL: velocidad rectilínea $\left(\mu \mathrm{m} \mathrm{s}^{-1}\right)$; VAP: velocidad promedio $\left(\mu \mathrm{m} \mathrm{s}^{-1}\right)$; LIN: índice de linealidad (\%); STR: índice de rectitud (\%); WOB: índice de oscilación (\%); ALH: desplazamiento lateral de la cabeza $(\mu \mathrm{m})$; $\mathrm{BCF}$ : frecuencia de entrecruzamiento $(\mathrm{Hz})$

El avance creciente en el desarrollo de esta tecnología y con la ayuda de mejoras en los hardware ha permitido incrementar la tasa de fotogramas, descrita como el número de imágenes capturadas por segundo $(16-60 \mathrm{~Hz})$ (Holt et al., 2007), contribuyendo así a mejorar la precisión en las estimaciones de los parámetros medidos con los sistemas asistidos de análisis seminal. La velocidad de fotogramas (Castellini et al., 2011; Valverde, et al., 2019e; Barquero et al., 2021), el número de imágenes (Contri et al., 2010) y la duración del tiempo de video de imagen (Valverde et al., 2019c) que un sistema pue- da capturar durante el análisis podrían afectar la distancia que un espermatozoide podría moverse entre imágenes sucesivas (Mortimer et al., 1988) y esto tiene un efecto directo sobre la trayectoria estimada de cada espermatozoide (Amann y Waberski, 2014).

Además, las trayectorias de los espermatozoides están relacionadas con el tamaño y el metabolismo del esperma (Tourmente et al., 2019) y varían dependiendo de la tasa de fotogramas utilizada (Mortimer y Swan, 1999). La primera imagen capturada en un sistema CASA-Mot identifica la posición del 
Cuadro 3. Análisis de regresión no lineal de variables de cinética espermática (medias $\pm \mathrm{EE}$ ) de verracos Pietrain medidas con diferentes profundidades de cámara de recuento ISAS ${ }^{\circledR} \mathrm{D} 4 \mathrm{C}(10,16,20 \mu \mathrm{m})$ considerando el nivel asintótico $(\alpha)$, tasa de incremento $(\beta)$ y correlación $\alpha / \beta\left(\rho^{\alpha, \beta}\right)$ necesarios para obtener un nivel umbral del $95 \%$ del valor máximo

\begin{tabular}{ccccccccc}
\hline Prof. & VCL & VSL & VAP & LIN & STR & WOB & ALH & BCF \\
\hline \multicolumn{7}{c}{ Valor asintótico $(\alpha)$; Modelo: $y=\beta * \alpha \exp (-\beta / x)$} \\
10 & $84.1 \pm 2.2$ & $45.6 \pm 1.7$ & $60.7 \pm 1.9$ & $73.9 \pm 1.7$ & $90.2 \pm 2.8$ & $79.7 \pm 1.7$ & $1.2 \pm 0.0$ & $19.8 \pm 0.7$ \\
16 & $86.5 \pm 1.8$ & $48.4 \pm 1.1$ & $52.3 \pm 1.1$ & $73.5 \pm 1.1$ & $93.3 \pm 0.9$ & $80.5 \pm 1.0$ & $1.1 \pm 0.0$ & $24.1 \pm 0.6$ \\
20 & $82.0 \pm 1.4$ & $40.8 \pm 0.8$ & $48.4 \pm 0.8$ & $79.7 \pm 1.0$ & $92.0 \pm 0.6$ & $83.5 \pm 0.8$ & $1.1 \pm 0.0$ & $21.6 \pm 0.4$ \\
\multicolumn{7}{c}{ Tasa de incremento $(\beta) ;$ Modelo: $y=\beta * \alpha \exp (-\beta / x)$} \\
10 & $17.8 \pm 0.9$ & $10.9 \pm 1.2$ & $15.4 \pm 1.0$ & $1.2 \pm 0.7$ & $4.8 \pm 0.9$ & $1.5 \pm 0.7$ & $-6.7 \pm 0.7$ & $26.3 \pm 1.2$ \\
16 & $14.0 \pm 0.7$ & $9.1 \pm 0.8$ & $7.4 \pm 0.7$ & $0.9 \pm 0.5$ & $2.3 \pm 0.3$ & $1.2 \pm 0.4$ & $-13.1 \pm 0.6$ & $28.5 \pm 0.8$ \\
20 & $7.7 \pm 0.5$ & $-0.1 \pm 0.7$ & $1.0 \pm 0.5$ & $2.7 \pm 0.4$ & $1.1 \pm 0.2$ & $2.2 \pm 0.3$ & $-15.7 \pm 0.5$ & $22.8 \pm 0.6$ \\
\hline
\end{tabular}

VCL: velocidad curvilínea $\left(\mu \mathrm{m} \mathrm{s}^{-1}\right)$; VSL: velocidad rectilínea $\left(\mu \mathrm{m} \mathrm{s}^{-1}\right)$; VAP: velocidad promedio $\left(\mu \mathrm{m} \mathrm{s}^{-1}\right)$; LIN: índice de linealidad (\%); STR: índice de rectitud (\%); WOB: índice de oscilación (\%); ALH: desplazamiento lateral de la cabeza $(\mu \mathrm{m})$; BCF: frecuencia de entrecruzamiento $(\mathrm{Hz})$

EE: error estándar. $(p<0.05)$

centroide de la cabeza de cada espermatozoide $\left(\mathrm{x}_{1}, \mathrm{y}_{1}\right)$ y conforme la célula se mueve en la secuencia de imágenes subsecuentes se vincula la nueva posición como $\left(\mathrm{x}_{2}, \mathrm{y}_{2}\right)$ en la segunda imagen, y así sucesivamente hasta la última imagen (Bompart et al., 2018).

Otras fuentes de variación consideradas en el presente estudio fueron el método para estimar las trayectorias de los espermatozoides, la concentración del semen (Lu et al., 2014) y también la profundidad de la cámara de recuento espermático (Bompart et al., 2018; Caldeira y Soler, 2018; Valverde $e t$ al., 2019a). En el presente estudio, con las cámaras de recuento utilizadas, se consideraron tres elementos que podrían estar relacionados con los resultados de los análisis; esto es, el diseño de la cámara (Valverde et $a l ., 2019$ a), la forma en que se carga la muestra dentro de la cámara (Gloria et al., 2013; Bompart et al., 2018) y la profundidad de esta (Valverde y Madrigal-Valverde, 2019).
En el presente estudio se analizaron cámaras desechables con siete campos longitudinales y llenado por efecto de la acción capilar. Todas las variables cinemáticas fueron mayores cuando hubo una mayor profundidad de cámara de recuento, lo cual podría explicarse por el efecto Segre-Silberberg (Douglas-Hamilton et al., 2005) debido a que en la mayoría de las cámaras disponibles en el mercado hay variaciones en el calibre del espacio de recuento, lo que favorece cambios en la distribución de los espermatozoides (Bompart et al., 2018; Valverde et al., 2019a). Históricamente, la profundidad de la cámara de recuento se relacionó con la calidad óptica y el aumento del objetivo del microscopio, por lo que la profundidad de $10 \mu \mathrm{m}$ era utilizada para lograr enfocar la mayor cantidad de células (Bompart et al., 2019). Al reducir el espacio de movilidad para el libre tránsito celular se altera el patrón de movilidad natural de los espermatozoides (Kraemer et al., 1998) y puede ser una limitante para la eva- 
Cuadro 4. Valores de las variables de cinética de los espermatozoides de verracos Pietrain en diferentes campos capturados a 25 fps en cámaras de recuento ISAS ${ }^{\circledR} \mathrm{D} 4 \mathrm{C}$ con profundidades de 10, 16 y $20 \mu \mathrm{m}$ (media \pm EE)

\begin{tabular}{|c|c|c|c|c|c|c|c|c|}
\hline $\begin{array}{l}\text { Campo } \\
\text { (n) }\end{array}$ & VCL & VSL & VAP & LIN & STR & WOB & ALH & $\mathrm{BCF}$ \\
\hline \multicolumn{9}{|c|}{$\mathrm{ISAS}^{\circledR} \mathrm{D} 4 \mathrm{C} 10$} \\
\hline $\begin{array}{c}\text { I } \\
(260)\end{array}$ & $41.1 \pm 0.8^{\mathrm{a}}$ & $29.3 \pm 0.9^{\mathrm{ab}}$ & $32.6 \pm 0.8^{\mathrm{ab}}$ & $69.7 \pm 1.3^{\mathrm{a}}$ & $75.3 \pm 2.0^{\mathrm{ab}}$ & $75.8 \pm 1.4^{\mathrm{ab}}$ & $1.5 \pm 0.0^{\mathrm{a}}$ & $6.7 \pm 0.2^{\mathrm{a}}$ \\
\hline $\begin{array}{c}\text { II } \\
(309)\end{array}$ & $40.7 \pm 0.7^{\mathrm{a}}$ & $31.0 \pm 0.8^{\mathrm{a}}$ & $33.6 \pm 0.7^{\mathrm{a}}$ & $74.8 \pm 1.3^{b}$ & $78.6 \pm 1.8^{\mathrm{a}}$ & $77.7 \pm 1.3^{\mathrm{a}}$ & $1.5 \pm 0.0^{\mathrm{a}}$ & $6.9 \pm 0.2^{\mathrm{a}}$ \\
\hline $\begin{array}{c}\text { III } \\
(493)\end{array}$ & $40.6 \pm 0.6^{\mathrm{a}}$ & $28.1 \pm 0.6^{\mathrm{b}}$ & $31.4 \pm 0.6^{\mathrm{b}}$ & $68.1 \pm 0.9^{\mathrm{a}}$ & $72.0 \pm 1.4^{\mathrm{b}}$ & $72.9 \pm 1.0^{\mathrm{b}}$ & $1.6 \pm 0.0^{\mathrm{b}}$ & $7.0 \pm 0.1^{\mathrm{a}}$ \\
\hline \multicolumn{9}{|c|}{ ISA ${ }^{\circledR} \mathrm{D} 4 \mathrm{C} 16$} \\
\hline $\begin{array}{c}\text { I } \\
(503)\end{array}$ & $50.2 \pm 0.7^{\mathrm{a}}$ & $32.8 \pm 0.6^{\mathrm{a}}$ & $38.7 \pm 0.6^{\mathrm{a}}$ & $71.7 \pm 0.8^{\mathrm{a}}$ & $84.4 \pm 0.8^{\mathrm{a}}$ & $77.6 \pm 0.7^{\mathrm{a}}$ & $1.8 \pm 0.0^{\mathrm{a}}$ & $7.8 \pm 0.1^{\mathrm{a}}$ \\
\hline $\begin{array}{c}\text { II } \\
(555)\end{array}$ & $50.8 \pm 0.7^{\mathrm{a}}$ & $33.9 \pm 0.6^{\mathrm{a}}$ & $39.1 \pm 0.6^{\mathrm{a}}$ & $70.4 \pm 0.8^{\mathrm{a}}$ & $85.6 \pm 0.7^{\mathrm{a}}$ & $77.4 \pm 0.6^{\mathrm{a}}$ & $1.8 \pm 0.0^{\mathrm{a}}$ & $8.4 \pm 0.1^{\mathrm{b}}$ \\
\hline $\begin{array}{c}\text { III } \\
(908)\end{array}$ & $50.4 \pm 0.5^{\mathrm{a}}$ & $33.3 \pm 0.5^{\mathrm{a}}$ & $38.4 \pm 0.5^{\mathrm{a}}$ & $70.9 \pm 0.6^{\mathrm{a}}$ & $85.5 \pm 0.6^{\mathrm{a}}$ & $76.3 \pm 0.5^{\mathrm{a}}$ & $1.8 \pm 0.0^{\mathrm{a}}$ & $8.5 \pm 0.1^{\mathrm{b}}$ \\
\hline \multicolumn{9}{|c|}{ ISAS ${ }^{\circledR} \mathrm{D} 4 \mathrm{C} 20$} \\
\hline $\begin{array}{c}\text { I } \\
(980)\end{array}$ & $61.3 \pm 0.2^{\mathrm{a}}$ & $40.7 \pm 0.3^{\mathrm{a}}$ & $47.1 \pm 0.3^{\mathrm{a}}$ & $71.7 \pm 0.5^{\mathrm{a}}$ & $86.6 \pm 0.4^{\mathrm{a}}$ & $76.9 \pm 0.4^{\mathrm{a}}$ & $2.1 \pm 0.0^{\mathrm{a}}$ & $8.7 \pm 0.1^{\mathrm{a}}$ \\
\hline $\begin{array}{c}\text { II } \\
(967)\end{array}$ & $61.1 \pm 0.2^{\mathrm{ab}}$ & $41.1 \pm 0.3^{\mathrm{a}}$ & $46.5 \pm 0.3^{\mathrm{ab}}$ & $71.3 \pm 0.5^{\mathrm{a}}$ & $88.3 \pm 0.4^{b}$ & $76.2 \pm 0.4^{\mathrm{a}}$ & $2.1 \pm 0.0^{\mathrm{a}}$ & $8.9 \pm 0.1^{\mathrm{ab}}$ \\
\hline $\begin{array}{c}\text { III } \\
(1501)\end{array}$ & $60.8 \pm 0.2^{\mathrm{b}}$ & $41.0 \pm 0.3^{\mathrm{a}}$ & $46.1 \pm 0.2^{b}$ & $72.1 \pm 0.4^{\mathrm{a}}$ & $88.9 \pm 0.3^{b}$ & $76.0 \pm 0.3^{\mathrm{a}}$ & $2.2 \pm 0.0^{\mathrm{a}}$ & $9.1 \pm 0.1^{\mathrm{b}}$ \\
\hline \multicolumn{9}{|c|}{ 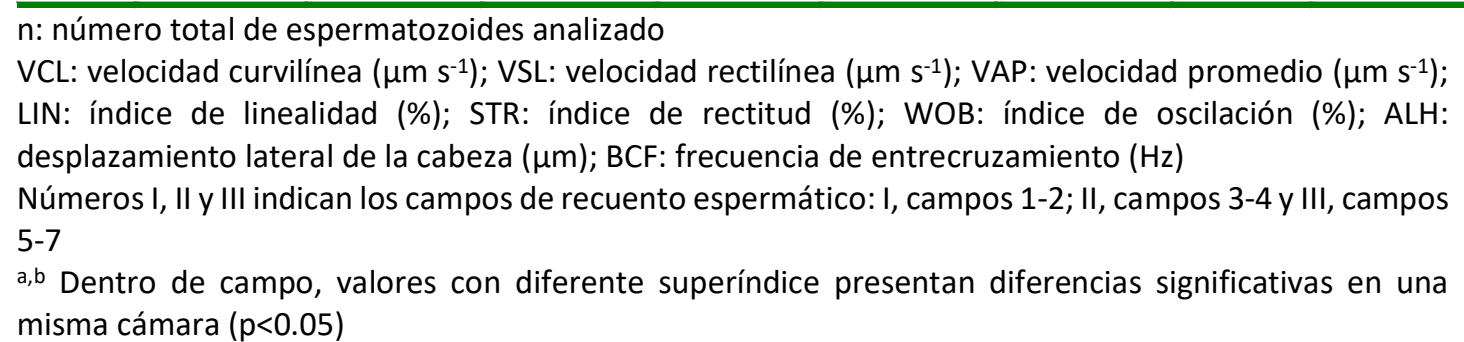 } \\
\hline
\end{tabular}

luación de los espermatozoides en condiciones de hiperactivación (Morales et al., 1988).

En el presente trabajo se encontraron diferencias en todas las variables cinemáticas de los espermatozoides conforme se incrementó la profundidad de la cámara. Estos resultados coinciden con trabajos en caprino para la VSL medida en cámaras de 20 y $10 \mu \mathrm{m}$
(Del Gallego et al., 2017) y en VCL, VAP, WOB, ALH y BCF en semen bovino al utilizar cámaras con $20 \mu \mathrm{m}$ de profundidad (Bompart et al., 2019). También se sugiere que la forma de carga por capilaridad es un punto de inflexión en la determinación del patrón de movilidad debido a que la acción capilar, como consecuencia del flujo de líquido resultante, podría afectar la cola de los 
Cuadro 5. Valores de las variables de cinética de los espermatozoides de verracos Pietrain en diferentes campos capturados a 40 fps en cámaras de recuento $I S A S^{\circledR} \mathrm{D} 4 \mathrm{C}$ con profundidades de 10, 16 y $20 \mu \mathrm{m}$ (media $\pm \mathrm{EE}$ )

\begin{tabular}{|c|c|c|c|c|c|c|c|c|}
\hline $\begin{array}{c}\text { Campo } \\
\text { (n) }\end{array}$ & VCL & VSL & VAP & LIN & STR & WOB & ALH & $\mathrm{BCF}$ \\
\hline \multicolumn{9}{|c|}{$\mathrm{ISAS}^{\circledR} \mathrm{D} 4 \mathrm{C} 10$} \\
\hline $\begin{array}{c}\text { I } \\
(142)\end{array}$ & $59.4 \pm 1.8^{\mathrm{a}}$ & $39.5 \pm 1.7^{\mathrm{a}}$ & $48.0 \pm 1.7^{\mathrm{a}}$ & $75.7 \pm 1.7^{\mathrm{a}}$ & $83.8 \pm 2.6^{\mathrm{a}}$ & $78.0 \pm 1.4^{\mathrm{a}}$ & $1.4 \pm 0.0^{\mathrm{a}}$ & $10.8 \pm 0.4^{\mathrm{a}}$ \\
\hline $\begin{array}{c}\text { II } \\
(156)\end{array}$ & $57.1 \pm 1.7^{\mathrm{ab}}$ & $33.8 \pm 1.6^{\mathrm{b}}$ & $44.5 \pm 1.6^{\mathrm{ab}}$ & $70.4 \pm 1.6^{\mathrm{b}}$ & $79.9 \pm 2.5^{\mathrm{a}}$ & $76.0 \pm 1.4^{\mathrm{a}}$ & $1.4 \pm 0.0^{\mathrm{a}}$ & $10.5 \pm 0.4^{\mathrm{a}}$ \\
\hline $\begin{array}{c}\text { III } \\
(222)\end{array}$ & $54.0 \pm 1.5^{\mathrm{b}}$ & $35.5 \pm 1.3^{\mathrm{ab}}$ & $41.6 \pm 1.4^{b}$ & $69.5 \pm 1.3^{b}$ & $76.9 \pm 2.1^{\mathrm{a}}$ & $76.4 \pm 1.2^{\mathrm{a}}$ & $1.4 \pm 0.0^{\mathrm{a}}$ & $10.1 \pm 0.4^{\mathrm{a}}$ \\
\hline \multicolumn{9}{|c|}{$\mathrm{ISAS}^{\circledR} \mathrm{D} 4 \mathrm{C} 16$} \\
\hline $\begin{array}{c}\text { I } \\
(374)\end{array}$ & $55.0 \pm 1.3^{\mathrm{a}}$ & $39.1 \pm 1.0^{\mathrm{a}}$ & $43.9 \pm 1.0^{\mathrm{a}}$ & $72.8 \pm 1.1^{\mathrm{a}}$ & $88.8 \pm 0.8^{a}$ & $80.0 \pm 0.9^{\mathrm{a}}$ & $1.4 \pm 0.0^{\mathrm{a}}$ & $9.7 \pm 0.3^{\mathrm{a}}$ \\
\hline $\begin{array}{c}\text { II } \\
(344)\end{array}$ & $56.6 \pm 1.3^{\mathrm{ab}}$ & $38.8 \pm 1.1^{\mathrm{a}}$ & $43.2 \pm 1.1^{\mathrm{a}}$ & $70.9 \pm 1.1^{\mathrm{a}}$ & $88.8 \pm 0.8^{\mathrm{a}}$ & $76.8 \pm 0.9^{b}$ & $1.5 \pm 0.0^{\mathrm{b}}$ & $10.0 \pm 0.3^{\mathrm{ab}}$ \\
\hline $\begin{array}{c}\text { III } \\
(459)\end{array}$ & $58.8 \pm 1.2^{\mathrm{b}}$ & $40.0 \pm 0.9^{\mathrm{a}}$ & $45.2 \pm 0.9^{\mathrm{a}}$ & $70.5 \pm 1.0^{\mathrm{a}}$ & $87.4 \pm 0.7^{\mathrm{a}}$ & $76.9 \pm 0.8^{\mathrm{b}}$ & $1.5 \pm 0.0^{\mathrm{b}}$ & $10.6 \pm 0.2^{b}$ \\
\hline \multicolumn{9}{|c|}{$\mathrm{ISAS}^{\circledR} \mathrm{D} 4 \mathrm{C} 20$} \\
\hline $\begin{array}{c}\text { I } \\
(822)\end{array}$ & $64.7 \pm 1.1^{\mathrm{a}}$ & $41.5 \pm 0.7^{\mathrm{a}}$ & $47.6 \pm 0.8^{\mathrm{a}}$ & $74.2 \pm 0.7^{\mathrm{a}}$ & $89.6 \pm 0.4^{\mathrm{a}}$ & $79.4 \pm 0.5^{\mathrm{a}}$ & $1.6 \pm 0.0^{\mathrm{a}}$ & $11.5 \pm 0.2^{\mathrm{a}}$ \\
\hline $\begin{array}{c}\text { II } \\
(674)\end{array}$ & $65.6 \pm 1.2^{\mathrm{a}}$ & $41.3 \pm 0.8^{\mathrm{a}}$ & $47.3 \pm 0.8^{\mathrm{a}}$ & $73.8 \pm 0.7^{\mathrm{a}}$ & $89.3 \pm 0.4^{\mathrm{a}}$ & $78.7 \pm 0.6^{\mathrm{a}}$ & $1.7 \pm 0.0^{\mathrm{a}}$ & $11.6 \pm 0.2^{\mathrm{a}}$ \\
\hline $\begin{array}{c}\text { III } \\
(1009)\end{array}$ & $64.4 \pm 1.0^{\mathrm{a}}$ & $39.9 \pm 0.7^{\mathrm{a}}$ & $46.1 \pm 0.7^{\mathrm{a}}$ & $73.4 \pm 0.6^{\mathrm{a}}$ & $88.8 \pm 0.4^{\mathrm{a}}$ & $78.6 \pm 0.5^{\mathrm{a}}$ & $1.6 \pm 0.0^{\mathrm{a}}$ & $11.4 \pm 0.2^{\mathrm{a}}$ \\
\hline
\end{tabular}

$\mathrm{n}$ : número total de espermatozoides analizado

VCL: velocidad curvilínea $\left(\mu \mathrm{m} \mathrm{s}^{-1}\right)$; VSL: velocidad rectilínea $\left(\mu \mathrm{m} \mathrm{s}^{-1}\right)$; VAP: velocidad promedio $\left(\mu \mathrm{m} \mathrm{s}^{-1}\right)$; LIN: índice de linealidad (\%); STR: índice de rectitud (\%); WOB: índice de oscilación (\%); ALH: desplazamiento lateral de la cabeza $(\mu \mathrm{m})$; BCF: frecuencia de entrecruzamiento $(\mathrm{Hz})$ Números I, II y III indican los campos de recuento espermático: I, campos 1-2; II, campos 3-4 y III, campos 5-7

a,b Dentro de campo, valores con diferente superíndice presentan diferencias significativas en una misma cámara $(p<0.05)$

espermatozoides y afectar el movimiento posterior (Lenz et al., 2011; Palacín et al., 2013).

En el análisis de regresión no lineal se estimó la tasa de fotogramas máxima necesaria para determinar el umbral asintótico en donde un incremento en la tasa de fotogramas no mejorará la precisión de la estimación de la trayectoria del espermatozoide. Este valor varió según el tipo de cámara de recuento, pero fue superior a $80 \mathrm{fps}$, definido como el valor de VCL al ser esta variable la más sensible al cambio con la tasa de fotogramas. Los resultados fueron similares a los descritos en semen equino y caprino por Hoogewijs et al. (2012) y Del Gallego et al. (2017), res- 
Cuadro 6. Valores de las variables de cinética de los espermatozoides de verracos Pietrain en diferentes campos capturados a $60 \mathrm{fps}$ en cámaras de recuento ISAS ${ }^{\circledR} \mathrm{D} 4 \mathrm{C}$ con profundidades de 10, 16 y $20 \mu \mathrm{m}$ (media $\pm \mathrm{DE}$ )

\begin{tabular}{|c|c|c|c|c|c|c|c|c|}
\hline $\begin{array}{l}\text { Campo } \\
\text { (n) }\end{array}$ & VCL & VSL & VAP & LIN & STR & WOB & ALH & $\mathrm{BCF}$ \\
\hline \multicolumn{9}{|c|}{ ISAS $^{\circledR} \mathrm{D} 4 \mathrm{C} 10$} \\
\hline $\begin{array}{c}\text { I } \\
(131)\end{array}$ & $63.1 \pm 2.0^{\mathrm{a}}$ & $36.3 \pm 1.6^{\mathrm{a}}$ & $48.2 \pm 1.7^{\mathrm{a}}$ & $75.1 \pm 1.5^{\mathrm{a}}$ & $85.4 \pm 2.3^{\mathrm{a}}$ & $79.6 \pm 1.9^{\mathrm{a}}$ & $1.3 \pm 0.0^{\mathrm{a}}$ & $12.6 \pm 0.6^{\mathrm{a}}$ \\
\hline $\begin{array}{c}\text { II } \\
(96)\end{array}$ & $57.1 \pm 2.4^{\mathrm{a}}$ & $37.2 \pm 1.8^{\mathrm{a}}$ & $40.9 \pm 2.0^{\mathrm{b}}$ & $70.8 \pm 1.7^{\mathrm{a}}$ & $83.6 \pm 2.6^{\mathrm{a}}$ & $76.6 \pm 1.8^{\mathrm{a}}$ & $1.2 \pm 0.0^{\mathrm{a}}$ & $12.7 \pm 0.7^{\mathrm{a}}$ \\
\hline $\begin{array}{l}\text { III } \\
(89)\end{array}$ & $59.4 \pm 2.5^{\mathrm{a}}$ & $37.0 \pm 1.9^{\mathrm{a}}$ & $42.8 \pm 2.1^{b}$ & $70.8 \pm 1.8^{\mathrm{a}}$ & $82.1 \pm 2.7^{\mathrm{a}}$ & $76.3 \pm 1.9^{\mathrm{a}}$ & $1.3 \pm 0.0^{\mathrm{a}}$ & $12.8 \pm 0.7^{\mathrm{a}}$ \\
\hline \multicolumn{9}{|c|}{ ISAS ${ }^{\circledR} \mathrm{D} 4 \mathrm{C} 16$} \\
\hline $\begin{array}{c}\text { I } \\
(187)\end{array}$ & $76.9 \pm 2.2^{\mathrm{a}}$ & $43.8 \pm 1.0^{\mathrm{a}}$ & $48.6 \pm 0.9^{a}$ & $73.8 \pm 1.4^{\mathrm{a}}$ & $90.3 \pm 0.7^{\mathrm{a}}$ & $80.1 \pm 1.3^{\mathrm{a}}$ & $1.5 \pm 0.0^{\mathrm{a}}$ & $16.5 \pm 0.5^{\mathrm{a}}$ \\
\hline $\begin{array}{c}\text { II } \\
(173)\end{array}$ & $73.7 \pm 2.3^{\mathrm{ab}}$ & $40.8 \pm 1.0^{\mathrm{b}}$ & $45.8 \pm 1.0^{\mathrm{b}}$ & $72.8 \pm 1.5^{\mathrm{a}}$ & $89.5 \pm 0.7^{\mathrm{a}}$ & $78.0 \pm 1.3^{\mathrm{a}}$ & $1.4 \pm 0.0^{\mathrm{ab}}$ & $17.4 \pm 0.5^{\mathrm{a}}$ \\
\hline $\begin{array}{c}\text { III } \\
(242)\end{array}$ & $69.3 \pm 2.0^{\mathrm{b}}$ & $37.8 \pm 0.8^{c}$ & $42.4 \pm 0.8^{c}$ & $72.7 \pm 1.2^{\mathrm{a}}$ & $89.7 \pm 0.6^{\mathrm{a}}$ & $79.8 \pm 1.1^{\mathrm{a}}$ & $1.4 \pm 0.0^{\mathrm{b}}$ & $16.4 \pm 0.5^{\mathrm{a}}$ \\
\hline \multicolumn{9}{|c|}{$\mathrm{ISAS}^{\circledR} \mathrm{D} 4 \mathrm{C} 20$} \\
\hline $\begin{array}{c}\text { I } \\
(173)\end{array}$ & $78.9 \pm 3.2^{\mathrm{a}}$ & $41.6 \pm 1.3^{\mathrm{a}}$ & $49.2 \pm 1.2^{\mathrm{a}}$ & $79.7 \pm 1.6^{a}$ & $91.6 \pm 0.7^{\mathrm{a}}$ & $78.5 \pm 1.5^{\mathrm{a}}$ & $1.6 \pm 0.1^{\mathrm{a}}$ & $16.7 \pm 0.6^{\mathrm{a}}$ \\
\hline $\begin{array}{c}\text { II } \\
(75)\end{array}$ & $83.4 \pm 4.8^{\mathrm{ab}}$ & $41.0 \pm 1.9^{\mathrm{a}}$ & $49.1 \pm 1.9^{\mathrm{a}}$ & $75.8 \pm 2.5^{\mathrm{a}}$ & $91.5 \pm 1.0^{\mathrm{a}}$ & $78.8 \pm 2.3^{\mathrm{a}}$ & $1.7 \pm 0.1^{\mathrm{a}}$ & $18.8 \pm 0.9^{\mathrm{b}}$ \\
\hline $\begin{array}{c}\text { III } \\
(116)\end{array}$ & $90.6 \pm 3.9^{b}$ & $41.3 \pm 1.6^{\mathrm{a}}$ & $47.6 \pm 1.5^{\mathrm{a}}$ & $81.3 \pm 2.0^{\mathrm{a}}$ & $91.5 \pm 0.8^{\mathrm{a}}$ & $84.8 \pm 1.8^{\mathrm{b}}$ & $1.7 \pm 0.1^{\mathrm{a}}$ & $17.3 \pm 0.7^{\mathrm{ab}}$ \\
\hline
\end{tabular}

n: número total de espermatozoides analizado

VCL: velocidad curvilínea $\left(\mu \mathrm{m} \mathrm{s}^{-1}\right)$; VSL: velocidad rectilínea $\left(\mu \mathrm{m} \mathrm{s}^{-1}\right)$; VAP: velocidad promedio $\left(\mu \mathrm{m} \mathrm{s}^{-1}\right)$; LIN: índice de linealidad (\%); STR: índice de rectitud (\%); WOB: índice de oscilación (\%); ALH: desplazamiento lateral de la cabeza $(\mu \mathrm{m})$; BCF: frecuencia de entrecruzamiento $(\mathrm{Hz})$

Números I, II y III indican los campos de recuento espermático: I, campos 1-2; II, campos 3-4 y III, campos 5-7

a,b,c Dentro de campo, valores con diferente superíndice presentan diferencias significativas en una misma cámara $(p<0.05)$

pectivamente, aunque ellos utilizaron una tasa de fotogramas menor a $25 \mathrm{fps}$. Por otro lado, Contri et al. (2010) no encontraron diferencias en los parámetros cinemáticos del semen de toros al utilizar 30 o 45 fotogramas por campo.

Al estudiar el efecto de la posición dentro de la cámara de recuento, los valores cinemáticos no fueron diferentes del lugar de deposición de la muestra; resultados que concuerdan con los descritos para bovino (Bompart et al., 2019), humano (Soler et al., 2012) y caprino (Del Gallego et al., 2017) utilizando cámaras de 10 y $20 \mu \mathrm{m}$, mientras en un estudio con semen de zorro se encontró una disminución de los valores cinemáticos entre el último campo con la primera parte de la cámara (Soler et al., 2014). Estos resultados podrían indicar que las diferencias 
encontradas no se relacionan con el diseño de la cámara, sino con diferencias entre especies.

En trabajos donde se estudió el efecto de la movilidad y cinemática a lo largo del área ecuatorial del portaobjetos mostraron diferencias en los campos cercanos a la frontera, pero no en otras áreas de muestreo (Nöthling y dos Santos, 2012). En el presente trabajo, considerando la tasa de fotogramas por campo y el tipo de cámara, se pudo observar diferencias en algunas variables para el campo analizado. Los valores más altos obtenidos cerca del lugar donde se depositó la gota (campo I) y los más bajos al final del movimiento del fluido (campo III) no podrían explicarse del todo por el flujo de Poiseuille y el consecuente efecto Segre-Silberberg (Kuster, 2005) o el posterior efecto de tensión superficial alrededor del cubreobjetos (Lenz et al., 2011), pudiendo ser solamente diferencias atribuibles a la especie animal.

Los resultados del presente estudio indican que con diferentes profundidades de cámara de recuento y tasa de fotogramas por campo no es posible traducir los resultados obtenidos con el uso de un tipo de cámara en resultados cuando se utiliza otro tipo de cámara. Esto confirma que la elección de la cámara de conteo tiene un efecto importante en los patrones de movilidad.

\section{ConClusiones}

- El uso de diferentes cámaras de recuento conduce a cambios significativos en la estimación de los parámetros cinemáticos de los espermatozoides de verraco.

- Una mayor frecuencia de fotogramas por segundo aporta mejor información para la estimación de las trayectorias, punto por punto, de los centroides de las células lo que incide directamente en los patrones cinemáticos del semen.
- Existe una variación importante a lo largo del área longitudinal de captura que es específica para cada tipo de cámara de recuento.

\section{Agradecimientos}

Los autores agradecen al Programa de Producción Agropecuaria (PPA) de la Escuela de Agronomía del Instituto Tecnológico de Costa Rica y al Ing. Wilfrido Paniagua Madrigal, por el apoyo recibido en la Granja «La Esmeralda» durante el desarrollo de la investigación.

\section{Literatura Citada}

1. Amann R, Waberski D. 2014. Computer-assisted sperm analysis (CASA): Capabilities and potential developments. Theriogenology 81: 5-17. doi: 10.1016/j.theriogenology.2013.09.004

2. Barquero V, Víquez, L, Calderón-calderón J, Valverde A. 2021. Frecuencia de fotogramas óptima para evaluar la cinética espermática de verracos con un sistema CASA-Mot. Agron Mesoam 32: 1-18. doi:10.15517/am.v32i1.41928.

3. Bompart D, García-Molina A, Valverde A, Caldeira C, Yániz J, Núñez de Murga M, Soler C. 2018. CASA-Mot technology: how results are affected by the frame rate and counting chamber. Reprod Fert Develop 30: 810819. doi: 10.1071/RD17551

4. Bompart D, Vázquez R, Gómez R, Valverde A, Roldán E, García-Molina $A$, Soler C. 2019. Combined effects of type and depth of counting chamber, and rate of image frame capture, on bull sperm motility and kinematics. Anim Reprod Sci 209: 106169. doi: 10.1016/ j.anireprosci.2019.106169

5. Boryshpolets S, Kowalski RK, Dietrich GJ, Dzyuba B, Ciereszko A. 2013. Different computer-assisted sperm 
analysis (CASA) systems highly influence sperm motility parameters. Theriogenology 80: 758-765. doi: 10.1016/ j.theriogenology.2013.06.019

6. Broekhuijse M, Gaustad A, Bolarin Guillén A, Knol E. 2015. Efficient boar semen production and genetic contribution: the impact of low-dose artificial insemination on fertility. Reprod Domest Anim 50: 103-109. doi: 10.1111/rda. 12558

7. Broekhuijse MLWJ, Šoštariae E, Feitsma H, Gadella BM. 2012. The value of microscopic semen motility assessment at collection for a commercial artificial insemination center, a retrospective study on factors explaining variation in pig fertility. Theriogenology 77: 1466-1479.e3. doi: 10.1016/j.theriogenology.2011.11.016

8. Caldeira C, Hernández-Ibáñez $S$, Valverde A, Martin P, HerranzJusdado JG, Gallego V, Asturiano JF, et al. 2019. Standardization of sperm motility analysis by using CASA-Mot for Atlantic salmon (Salmo salar), European eel (Anguilla anguilla) and Siberian sturgeon (Acipenser baerii). Aquaculture 502: 223-231. doi: 10.1016/ j.aquaculture.2018.12.001

9. Caldeira C, Soler C. 2018. Fish sperm assessment using software and cooling devices. Jove-J Vis Exp 137: e56823. doi: $10.3791 / 56823$

10. Castellini C, Dal Bosco A, Ruggeri S, Collodel G. 2011. What is the best frame rate for evaluation of sperm motility in different species by computer-assisted sperm analysis? Fertil Steril 96: 24-27. doi: 10.1016/j.fertnstert.2011.-04.096

11. Contri A, Valorz C, Faustini M, Wegher L, Carluccio A. 2010. Effect of semen preparation on casa motility results in cryopreserved bull spermatozoa. Theriogenology 74: 424-435. doi: 10.1016/ J.theriogenology.2010.02.025

12. Douglas-Hamilton DH, Smith NG, Kuster CE, Vermeiden JPW, Althouse $G C .2005$. Capillary-loaded particle fluid dynamics: effect on estimation of sperm concentration. J Androl 26: 115-122.
13. Ehlers J, Behr M, Bollwein H, Beyerbach M, Waberski D. 2011. Standardization of computer-assisted semen analysis using an e-learning application. Theriogenology 76: 448-454. doi: 10.1016/j.theriogenology.2011.02.021

14. Gacem S, Bompart D, Valverde A, Catalán J, Miró J, Soler C. $2020 a$. Optimal frame rate when there were stallion sperm motility evaluations and determinations for kinematic variables using CASA-Mot analysis in different counting chambers. Anim Reprod Sci 223. doi: 10.1016/j.anireprosci.2020.106643

15. Gacem S, Catalán J, Valverde A, Soler C, Miró J. 2020b. Optimization of CASA-mot analysis of donkey sperm: optimum frame rate and values of kinematic variables for different counting chamber and fields. Animals 10: 1993. doi: 10.3390/ani10111993

16. Gallagher MT, Smith DJ, KirkmanBrown JC. 2018. CASA: tracking the past and plotting the future. Reprod Fert Develop 30: 867-874. doi: 10.1071/ RD17420

17. Del Gallego R, Sadeghi S, Blasco E, Soler C, Yániz J, Silvestre M. 2017. Effect of chamber characteristics, loading and analysis time on motility and kinetic variables analysed with the CASA-mot system in goat sperm. Anim Reprod Sci 177: 97-104. doi: 10.1016/ j.anireprosci.2016.12.010

18. Di Rienzo JA, Casanoves F, Balzarini MG, Gonzalez L, Tablada M, Robledo C. 2020. InfoStat version 2020. http:// www.infostat.com.ar

19. Gloria A, Carluccio A, Contri A, Wegher L, Valorz C, Robbe D. 2013. The effect of the chamber on kinetic results in cryopreserved bull spermatozoa. Andrology 1: 879-885. doi: 10.1111/ j.2047-2927.2013.00121.x

20. Holt W, O'Brien J, Abaigar T. 2007. Applications and interpretation of computer-assisted sperm analyses and sperm sorting methods in assisted 
breeding and comparative research. Reprod Fert Develop 19: 709-718.

21. Hoogewijs MK, De Vliegher SP, Govaere JL, De Schauwer C, De Kruif $A$, Van Soom A. 2012. Influence of counting chamber type on CASA outcomes of equine semen analysis. Equine Vet J 44: 542-549. doi: 10.1111/ j.2042-3306.2011.00523.x

22. Kraemer M, Fillion C, Martin-Pont B, Auger J. 1998. Factors influencing human sperm kinematic measurements by the Celltrak computer-assisted sperm analysis system. Hum Reprod 13: 611619. doi: 10.1093/humrep/13.3.611

23. Kuster C. 2005. Sperm concentration determination between hemacytometric and CASA systems: Why they can be different. Theriogenology 64: 614-617. doi: 10.1016/j.theriogenology.2005.05.047

24. Lenz RW, Kjelland ME, VonderHaar K, Swannack TM, Moreno JF. 2011. A comparison of bovine seminal quality assessments using different viewing chambers with a computer-assisted semen analyzer. J Anim Sci 89: 383-388. doi: $10.2527 /$ jas.2010-3056

25. Lopez-Rodriguez A, Soom A Van, Arsenakis I, Maes D. 2017. Boar management and semen handling factors affect the quality of boar extended semen. Porcine Health Manag 3: 15. doi: 10.1186/s40813-017-0062-5

26. Lu JC, Huang YF, Lü NQ. 2014. Computer-aided sperm analysis: Past, present and future. Andrologia 46: 329338. doi: 10.1111/and.12093

27. Morales P, Overstreet JW, Katz DF. 1988. Changes in human sperm motion during capacitation in vitro. Reproduction 83: 119-128. doi: 10.1530/jrf.0.0830119

28. Mortimer D, Serres C, Mortimer S, Jouannet P. 1988. Influence of image sampling frequency on the perceived movement characteristics of progressively motile human spermatozoa. Gamete Res 20:313-327. doi: 10.1002/mrd.1120200307
29. Mortimer S, Swan M. 1999. Effect of image sampling frequency on established and smoothing-independent kinematic values of capacitating human spermatozoa. Hum Reprod 14: 997-1004. doi: 10.1093/humrep/14.4.997

30. National Research Council. 2012. Nutrient requirements of swine. Washington DC: The National Academies Press.

31. Nöthling JO, dos Santos IP. 2012. Which fields under a coverslip should one assess to estimate sperm motility? Theriogenology 77: 1686-1697. doi: 10.1016/j.theriogenology.2011.12.014

32. Palacín I, Vicente-Fiel S, Santolaria P, Yániz J. 2013. Standardization of CASA sperm motility assessment in the ram. Small Ruminant Res 112: 128-135. doi: 10.1016/j.smallrumres.2012.12.014

33. Soler C, Fuentes M, Sancho M, García A, Núñez de Murga M, Núñez de Murga J. 2012. Effect of counting chamber on seminal parameters, analyzing with the ISASv1 ${ }^{\circledR}$. Rev Int Androl 10: 132-138. doi: 10.1016/S1698-031X(12)70069-9

34. Soler C, García A, Contell J, Segervall J, Sancho M. 2014. Kinematics and subpopulations' structure definition of blue fox (Alopex lagopus) Sperm motility using the ISAS ${ }^{\circledR}$ V1 CASA system. Reprod Domest Anim 49: 560567. doi: 10.1111/rda.12310

35. Soler C, Picazo-Bueno J, Micó V, Valverde A, Bompart D, Blasco FJ, Alvarez JG, et al. 2018. Effect of counting chamber depth on the accuracy of lensless microscopy for the assessment of boar sperm motility. Reprod Fert Develop 30: 924-934. doi: 10.1071/RD17467

36. Soler C, Valverde A, Bompart D, Fereidounfar S, Sancho M, Yániz J, Garcia-Molina A, et al. 2017. New methods of semen analysis by casa. Agric Biol 52: 232-241. doi: 10.15389/ agrobiology.2017.2.232eng

37. Tourmente M, Varea-Sánchez M, Roldan ERS. 2019. Faster and more efficient swimming: energy consumption 
of murine spermatozoa under sperm competition. Biol Reprod 100: 420-428. doi: 10.1093/biolre/ioy197

38. Urbano L, Masson P, VerMilyea M, Kam M. 2017. Automatic tracking and motility analysis of human sperm in timelapse images. IEEE Trans Med Imaging 36: 792-801. doi: 10.1109/TMI.2016.2630720

39. Valverde A, Madrigal-Valverde M. 2018. Sistemas de análisis computadorizado de semen en la reproducción animal. Agron Mesoam 29: 469-484. doi: 10.15517/ma.v29i2.30613

40. Valverde A, Madrigal-Valverde M. 2019. Evaluación de cámaras de recuento sobre parámetros espermáticos de verracos analizados con un sistema CASA-Mot. Agron Mesoam 30: 447458. doi: 10.15517/am.v30i1.34145

41. Valverde A, Areán H., Fernández A, Bompart D, García-Molina A, LópezViana J, Soler C. 2019a. Combined effect of type and capture area of counting chamber and diluent on Holstein bull sperm kinematics. Andrologia 51: e13223. doi:10.1111/and.13223

42. Valverde A, Castro-Morales $\mathrm{O}, \mathrm{Madri-}$ gal-Valverde M, Soler C. $2019 b$. Sperm kinematics and morphometric subpopulations analysis with CASA systems: A review. Rev Biol Trop 67: 1473-1487.doi:10.15517/rbt.v67i6.35151

43. Valverde A, Madrigal-Valverde $M$, Lotz J, Bompart D, Soler C. 2019c. Effect of video capture time on sperm kinematic parameters in breeding boars. Livest Sci 220: 52-56. doi: 10.1016/ j.livsci.2018.12.008

44. Valverde A, Madrigal-Valverde M, Solís-Arias J, Paniagua-Madrigal W. 2019d. Variabilidad en los métodos de estimación de la concentración espermática en verracos. Agron Costarric 43: 25-43. doi: 10.15517/rac.v43i2.37793

45. Valverde A, Madrigal M, Caldeira $C$, Bompart D, de Murga JN, Arnau S, Soler C. 2019e. Effect of frame rate capture frequency on sperm kinematic parameters and subpopulation structure definition in boars, analysed with a CASA-Mot system. Reprod Domest Anim 54: 167-175. doi: 10.1111/rda. 13320

46. Valverde A, Barquero V, Soler C. 2020. The application of computer-assisted semen analysis (CASA) technology to optimise semen evaluation. A review. J Anim Feed Sci 29: 189-198. doi: 10.22358/JAFS/127691/2020 